\title{
Observational signature of the logarithmic terms in the soft-graviton theorem
}

\author{
Alok Laddha ${ }^{1, *}$ and Ashoke Sen ${ }^{2, \dagger}$ \\ ${ }^{1}$ Chennai Mathematical Institute, Siruseri, Chennai 603103, India \\ ${ }^{2}$ Harish-Chandra Research Institute, HBNI, Chhatnag Road, Jhusi, Allahabad 211019, India
}

(Received 8 November 2018; published 8 July 2019)

\begin{abstract}
We show that the recently discovered logarithmic terms in the soft-graviton theorem induce a late time component in the gravitational waveform that falls off as inverse power of time, producing a tail term to the linear memory effect.
\end{abstract}

DOI: 10.1103/PhysRevD.100.024009

One of the reasons for the recent interest in the softgraviton theorem is its connection to the memory effect $[1-4]$ - the fact that a passing gravitational wave causes a permanent change in the distance between two detectors placed in its path [5-8]. This connection usually proceeds via asymptotic symmetries [9-11] and has led to the prediction of a new kind of memory effect associated with the super-rotation symmetry [2]. In contrast, in Ref. [12], we established a direct connection between the soft factors that arise in soft theorems and the low frequency classical radiation in a classical process by taking the classical limit of the quantum scattering process. This has the advantage of being valid in all space-time dimensions, irrespective of whether or not the soft theorem can be related to an asymptotic symmetry. However, while applying this formula to four dimensions, we encounter a new phenomenon: due to the long range force on the particles involved in the scattering, the soft factor at the subleading order gets a contribution proportional to the logarithm of the energy of the soft radiation [13]. Our goal in this paper is to describe the observational signature of this logarithmic term in the soft-graviton theorem. We shall use $\hbar=c=8 \pi G=1$ units, although since we are analyzing classical radiation, $\hbar$ never enters any formula.

The result of this paper may be summarized as follows. In any process that involves the breakup of a massive object of mass $M$ into another object of mass $M_{0} \simeq M$ and a set of light particles, the gravitational waveform $e_{i j}^{T T}$ near future null infinity is given, at late

\footnotetext{
*aladdha@cmi.ac.in

†sen@hri.res.in
}

Published by the American Physical Society under the terms of the Creative Commons Attribution 4.0 International license. Further distribution of this work must maintain attribution to the author(s) and the published article's title, journal citation, and DOI. Funded by SCOAP ${ }^{3}$. retarded time $u$, by Eq. (7). $A_{i j}$ and $B_{i j}$ in this equation are determined in terms of the final state mass and velocity distribution of the light particles via Eqs. (11) and (12). The coefficient $A_{i j}$ describes the standard memory effect, while the coefficient $B_{i j}$ describes a tail term that falls off as an inverse power of $u$.

The setup we shall investigate is a process in which a system of mass $M$, describing the initial system, makes a transition into a system of mass $M_{0}<M$ and some matter/radiation that escapes the system. We shall work in the rest frame of the original system and assume for simplicity that the total energy carried by the escaping matter/radiation is small compared to the mass of the original system so that $M_{0} \simeq M$ and the recoil velocity of the final system is small [14]. An example of this would be the merger of neutron stars where a large amount of matter is ejected from the parent system but the total amount of energy lost is still small compared to the mass of the system that remains behind. Another example would be the merger of two black holes where the energy is radiated away gravitationally, but we shall see that the effects we shall describe vanish in the case where only massless particles carry away the energy. Our focus will be on the low frequency radiative component of the metric field $h_{\mu \nu} \equiv\left(g_{\mu \nu}-\eta_{\mu \nu}\right) / 2$.

In Ref. [13], a formula for the soft radiation was found in a situation where a light particle of mass $m$ scatters from a heavy particle of mass $M_{0}$. Here, a light particle refers to a particle carrying energy $\ll M_{0}$. However, since the softgraviton theorem expresses the result as independent sums over initial and final states, the result can be easily generalized to the case where there are no light particles in the initial state and multiple light particles in the final state. We shall now state this result. For more details, we refer the reader to Ref. [13].

Let $t_{0} \simeq|\vec{x}|+M_{0} \ln |\vec{x}| / 4 \pi$ be the time at which the peak of the gravitational radiation reaches the observer at $\vec{x}$. For the radiative part of the trace reversed metric 


$$
\begin{aligned}
\tilde{e}_{\mu \nu}(\omega, \vec{x}) & \equiv \int d t e^{i \omega\left(t-t_{0}\right)} e_{\mu \nu}(t, \vec{x}), \\
e_{\mu \nu}(t, \vec{x}) & \equiv\left\{h_{\mu \nu}(t, \vec{x})-\frac{1}{2} h_{\rho}^{\rho}(t, \vec{x}) \eta_{\mu \nu}\right\},
\end{aligned}
$$

the results of Refs. $[12,13]$, when applied to the situation where we have light particles only in the final state, can be stated as follows. Up to an overall constant phase that can be absorbed into a shift of the time coordinate, we have [16]

$$
\tilde{e}_{i j}(\omega, \vec{x})=\frac{i}{4 \pi|\vec{x}|} e^{-i M_{0} \omega \ln \omega^{-1} /(4 \pi)}\left[\sum_{a} m_{a} \beta_{a i} \beta_{a j} \frac{1}{1-\hat{n} \cdot \vec{\beta}_{a}} \frac{1}{\sqrt{1-\vec{\beta}_{a}^{2}}}\left\{\omega^{-1}+i C_{a} \ln \omega^{-1}+\text { finite }\right\}\right],
$$

where "finite" refers to terms that have a finite $\omega \rightarrow 0$ limit, the sum over $a$ runs over all the light final states, $\vec{\beta}_{a}$ is the velocity of the $a$ th light particle, $m_{a}$ is the mass of the $a$ th light particle, and

$$
C_{a} \equiv-M_{0} \frac{1-3 \vec{\beta}_{a}^{2}}{8 \pi\left|\vec{\beta}_{a}\right|^{3}}, \quad \hat{n} \equiv \frac{\vec{x}}{|\vec{x}|}, \quad k \equiv-\omega(1, \hat{n}) .
$$

The $\tilde{e}_{0 \mu}$ components are undetermined at this stage but can in principle be determined by using the constraint $k^{\mu} \tilde{e}_{\mu \nu}=0$ that follows from the linearized Einstein's equation. The result for $\tilde{e}_{\mu \nu}$ is ambiguous up to the linearized gauge transformation $\delta \tilde{e}_{\mu \nu}=\zeta_{\mu} k_{\nu}+\zeta_{\nu} k_{\mu}-\zeta . k \eta_{\mu \nu}$ for any 4-vector $\zeta$.

We now define the transverse traceless component $\tilde{e}_{i j}^{T T}$ as

$$
\tilde{e}_{i j}^{T T}=\tilde{e}_{i j}+\xi_{i} k_{j}+\xi_{j} k_{i}-\xi \delta_{i j},
$$

where the 3 -vector $\xi_{i}$ and the scalar $\xi$ are to be chosen such that

$$
k^{i} \tilde{e}_{i j}^{T T}=0, \quad \delta^{i j} \tilde{e}_{i j}^{T T}=0 .
$$

It is easy to see that $\tilde{e}_{i j}^{T T}$ is invariant under a gauge transformation. Using (2), (4), and (5), we now get, after expanding the exponential factor in (2) to the first subleading order,

$$
\begin{aligned}
& \tilde{e}_{i j}^{T T}(\omega, \vec{x})=\frac{i}{4 \pi|\vec{x}|} \sum_{a}\left\{\omega^{-1}+i\left(C_{a}-\frac{M_{0}}{4 \pi}\right) \ln \omega^{-1}+\text { finite }\right\} m_{a} \frac{1}{1-\hat{n} \cdot \vec{\beta}_{a}} \frac{1}{\sqrt{1-\vec{\beta}_{a}^{2}}}\left(\beta_{a i} \beta_{a j}\right)^{T T}, \\
& \left(\beta_{a i} \beta_{a j}\right)^{T T} \equiv\left\{\beta_{a i} \beta_{a j}-\hat{n} . \vec{\beta}_{a}\left(\hat{n}_{i} \beta_{a j}+\hat{n}_{j} \beta_{a i}\right)+\frac{1}{2}\left(\vec{\beta}_{a}^{2}+\left(\hat{n} \cdot \vec{\beta}_{a}\right)^{2}\right) \hat{n}_{i} \hat{n}_{j}+\frac{1}{2}\left(\left(\hat{n} \cdot \vec{\beta}_{a}\right)^{2}-\vec{\beta}_{a}^{2}\right) \delta_{i j}\right\} .
\end{aligned}
$$

The coefficient of $\ln \omega^{-1}$ proportional to $C_{a}$ represents the effect of late time radiation from the outgoing particles accelerating in the background gravitational field, while the term proportional to $M_{0} / 4 \pi$ represents the effect of backscattering of the soft graviton due to the background gravitational field [17-19].

Since (6) gives the small $\omega$ behavior of $\tilde{e}_{i j}^{T T}(\omega, \vec{x})$, it encodes the behavior of its inverse Fourier transform $e_{i j}^{T T}(t, \vec{x})$ at large time. We shall now explicitly find this behavior. Let us suppose that $e_{i j}^{T T}$ has the following asymptotic behavior,

$$
\begin{aligned}
e_{i j}^{T T}(t, \vec{x}) & \simeq\left\{\begin{array}{ll}
0 & \text { for } u \rightarrow-\infty \\
A_{i j}+u^{-1} B_{i j}+\mathcal{O}\left(u^{-2}\right) & \text { for } u \rightarrow \infty
\end{array},\right. \\
u & \equiv t-t_{0},
\end{aligned}
$$

where we allow logarithmic multiplicative factors in the $\mathcal{O}\left(u^{-2}\right)$ terms. As mentioned earlier, $t_{0}$ denotes the time when the peak of the gravitational wave reaches the observer at $\vec{x}$, but the precise choice is not important since a finite shift will not affect the expansion coefficients $A_{i j}$ and $B_{i j}$. Since $e_{i j}^{T T}(t, \vec{x})$ does not fall off as $u \rightarrow \infty$, the correct way to interpret (1) is to write $e^{i \omega u}=(i \omega)^{-1} \frac{d}{d u} e^{i \omega u}$ in the Fourier integral and carry out an integration by parts, ignoring the boundary terms. This gives

$$
\tilde{e}_{i j}^{T T}(\omega, \vec{x})=-\frac{1}{i \omega} \int d u e^{i \omega u} \partial_{u} e_{i j}^{T T}(t, \vec{x})
$$

We now express this as

$$
\begin{aligned}
\tilde{e}_{i j}^{T T}(\omega, \vec{x})= & -\frac{1}{i \omega} \int d u \partial_{u} e_{i j}^{T T}(t, \vec{x}) \\
& -\frac{1}{i \omega} \int d u\left\{e^{i \omega u}-1\right\} \partial_{u} e_{i j}^{T T}(t, \vec{x}) .
\end{aligned}
$$

The first term gives $i \omega^{-1} A_{i j}$. The second term can be estimated by dividing the integration region to $u \ll \epsilon^{-1}$, $\epsilon^{-1}<u<\eta \omega^{-1}, \quad \eta \omega^{-1}<u<\omega^{-1}$, and $\omega^{-1}<u<\infty$ 
where $\epsilon$ and $\eta$ are two small but finite positive numbers. Since $e^{i \omega u}-1$ is bounded in magnitude by $\omega u$ and since, for large negative $u, e_{i j}^{T T}(u, \vec{x})$ falls off fast, the contribution to $\tilde{e}_{i j}^{T T}$ from the $u<\epsilon^{-1}$ region remains finite in the $\omega \rightarrow 0$ limit. In the region $\epsilon^{-1}<u<\eta \omega^{-1}$, we can approximate $e^{i \omega u}-1$ as $i \omega u$ and $\partial_{u} e_{i j}^{T T}$ by $-B_{i j} / u^{2}$. Therefore, the integral gets a contribution of order $B_{i j}\left\{\ln \omega^{-1}+\ln (\eta \epsilon)\right\}$. In the region $\eta \omega^{-1}<u<\omega^{-1}$, we can use the results $\left|e^{i \omega u}-1\right| \leq \omega u$ and $\partial_{u} e_{i j}^{T T} \simeq-B_{i j} / u^{2}$ to argue that the integral is bounded in magnitude by $\ln (1 / \eta)$. Finally, in the region $u>\omega^{-1}$, we can use the results $\left|e^{i \omega u}-1\right| \leq 2$ and $\partial_{u} e_{i j}^{T T} \simeq-B_{i j} / u^{2}$ to show that the integral is bounded in magnitude by $2 B_{i j}$. Therefore, by taking $\epsilon, \eta$ to be small but fixed, we see that the leading contribution to the integral for small $\omega$ is given by

$$
\tilde{e}_{i j}^{T T}(\omega, \vec{x})=i \omega^{-1} A_{i j}+B_{i j} \ln \omega^{-1}+\text { finite. }
$$

Comparing (6) and (10) and using (3), we can determine the coefficients $A_{i j}$ and $B_{i j}$,

$$
\begin{aligned}
A_{i j}= & \frac{1}{4 \pi|\vec{x}|} \sum_{a} m_{a} \frac{1}{1-\hat{n} \cdot \vec{\beta}_{a}} \frac{1}{\sqrt{1-\vec{\beta}_{a}^{2}}}\left(\beta_{a i} \beta_{a j}\right)^{T T} \\
= & \frac{2 G}{|\vec{x}|} \sum_{a} m_{a} \frac{1}{1-\hat{n} \cdot \vec{\beta}_{a}} \frac{1}{\sqrt{1-\vec{\beta}_{a}^{2}}}\left(\beta_{a i} \beta_{a j}\right)^{T T} \\
B_{i j}= & \frac{M_{0}}{32 \pi^{2}|\vec{x}|} \sum_{a} m_{a} \frac{1}{1-\hat{n} \cdot \vec{\beta}_{a}} \\
& \times \frac{1}{\sqrt{1-\vec{\beta}_{a}^{2}}} \frac{1-3 \vec{\beta}_{a}^{2}+2\left|\vec{\beta}_{a}\right|^{3}}{\left|\vec{\beta}_{a}\right|^{3}}\left(\beta_{a i} \beta_{a j}\right)^{T T} \\
= & \frac{2 G^{2} M_{0}}{|\vec{x}|} \sum_{a} m_{a} \frac{1}{1-\hat{n}_{a} \cdot \vec{\beta}_{a}} \frac{1}{\sqrt{1-\vec{\beta}_{a}^{2}}} \\
& \times \frac{1-3 \vec{\beta}_{a}^{2}+2\left|\vec{\beta}_{a}\right|^{3}}{\left|\vec{\beta}_{a}\right|^{3}}\left(\beta_{a i} \beta_{a j}\right)^{T T},
\end{aligned}
$$

where in the last steps we have rewritten the result in terms of the Newton's constant $G=1 / 8 \pi$.

A term in $h_{i j}$ of the form $B_{i j} / u$ produces Riemann tensor components $R_{\text {uiuj }} \propto B_{i j} / u^{3}$. The associated Ricci tensor vanishes, showing that it satisfies vacuum Einstein's equation. On the other hand, the nonvanishing Riemann tensor shows that the result is not a gauge artifact. This has to be contrasted to the memory term $A_{i j}$ for which the Riemann tensor vanishes even though the effect is physical.

If we consider the case where all the final states are massless/ultrarelativistic particles for which $\left|\vec{\beta}_{a}\right|=1$, then we see from (12) that $B_{i j}$ vanishes. Therefore, in this case, there will be no tail effect-showing that the nonlinear memory effect [20-24] has no tail term of order $1 / u$. This shows that, in order to realize the tail effect, we need to focus on processes where some of the final state light particles are massive. For small $\left|\vec{\beta}_{a}\right|$, the tail term appears to dominate over the memory term -in fact, Eq. (12) gives the impression that $B_{i j}$ diverges as $\left|\vec{\beta}_{a}\right| \rightarrow 0$. However, we note that there is no real singularity in the $\left|\vec{\beta}_{a}\right| \rightarrow 0$ limit since it will take a period of order $G M_{0} /\left|\vec{\beta}_{a}\right|^{3}$ for the kinetic energy $m_{a} \vec{\beta}_{a}^{2} / 2$ of the particles to dominate the potential energy $G M_{0} m_{a} /\left(\left|\vec{\beta}_{a}\right| u\right)$ so that we can use the asymptotic formula for the particle trajectory used in deriving (2). After waiting for time $u \sim G M_{0} /\left|\vec{\beta}_{a}\right|^{3}$ after the peak of the gravitational wave has passed, the tail term $B_{i j} / u$ already becomes of the order of the memory term $A_{i j}$. It falls below the memory term as $u$ increases further.

The tail effect has been discussed earlier in various contexts, e.g., in Refs. [25-32]. Like us, the authors also explore the behavior of the gravitational waveform at late retarded time. However, since these papers do not have massive particles in the final state, their results cannot be directly compared to the one described here. To the best of our knowledge, the tail effect of the kind we are discussing first appeared in Ref. [33], and our results are in perfect agreement with the results of this paper [13]. In Ref. [13], we have also verified that our formula correctly reproduces the gravitational radiation during a scattering where the main force responsible for the scattering is electromagnetic instead of gravitational. The main new feature of our result is that the soft-graviton theorem provides a way to express the tail term in a compact form in terms of the velocity and mass distribution of the outgoing particles, without knowing the details of the scattering process.

The following simple calculation allows us to get an idea of the order of magnitude of this effect. Consider for example a core-collapse supernova [34] somewhere in our Galaxy that produces a neutron star of mass $M_{0} \sim M_{\odot}$, moving at a speed of $1000 \mathrm{~km} / \mathrm{s}$, and the momentum is balanced by ejected matter of total mass $m \sim M_{\odot} / 5$, moving in the opposite direction at a speed of about $5000 \mathrm{~km} / \mathrm{s}$. In this case, a rough estimate shows that the timescale $G M_{0} /\left|\beta_{a}\right|^{3}$ at which the tail effect becomes visible is of the order of a second and the amplitude $B_{i j} / u \sim G m|\beta|^{2} /|\vec{x}|$ of $h_{i j}$, at this time, is of order $10^{-22}$ [35]. These are at the edge of LIGO detection limits [36]. With improved detectors, such effects may be detectable even for supernova explosions outside our Galaxy. Therefore, it is not inconceivable that such effects may be observed in the near future.

We would like to thank P. Ajith, K. G. Arun, Rishabh Batra, Luc Blanchet, Miguel Campiglia, Thibault Damour, Bala Iyer, Ira Rothstein, Walter Goldberger, and Biswajit Sahoo for useful discussions. A. S. would like to thank the Abdus Salam International Centre for Theoretical 
Physics for hospitality during the final stages of this work. A. L. would like to thank International Center for Theoretical Sciences for their hospitality during the final stages of the work. The work of A. S. was supported in part by the J. C. Bose fellowship of the Department of Science and Technology, India.
[1] A. Strominger and A. Zhiboedov, Gravitational memory, BMS supertranslations and soft theorems, J. High Energy Phys. 01 (2016) 086.

[2] S. Pasterski, A. Strominger, and A. Zhiboedov, New gravitational memories, J. High Energy Phys. 12 (2016) 053.

[3] D. Kapec, V. Lysov, S. Pasterski, and A. Strominger, Higher-dimensional supertranslations and Weinberg's soft graviton theorem, Ann. Math. Sci. Appl. 2, 69 (2017).

[4] M. Pate, A. M. Raclariu, and A. Strominger, Gravitational memory in higher dimensions, J. High Energy Phys. 06 (2018) 138.

[5] Ya. B. Zeldovich and A. G. Polnarev, Sov. Astron. 18, 17 (1974).

[6] V. B. Braginsky and L. P. Grishchuk, Kinematic resonance and memory effect in free mass gravitational antennas, $\mathrm{Zh}$. Eksp. Teor. Fiz. 89, 744 (1985) [Sov. Phys. JETP 62, 427 (1985)].

[7] V. B. Braginsky and K. S. Thorne, Gravitational-wave bursts with memory and experimental prospects, Nature (London) 327, 123 (1987).

[8] M. Ludvigsen, Geodesic deviation at null infinity and the physical effects of very long wave gravitational radiation, Gen. Relativ. Gravit. 21, 1205 (1989).

[9] A. Strominger, On BMS invariance of gravitational scattering, J. High Energy Phys. 07 (2014) 152.

[10] T. He, V. Lysov, P. Mitra, and A. Strominger, BMS supertranslations and Weinbergs soft graviton theorem, J. High Energy Phys. 05 (2015) 151.

[11] M. Campiglia and A. Laddha, Asymptotic symmetries and subleading soft graviton theorem, Phys. Rev. D 90, 124028 (2014).

[12] A. Laddha and A. Sen, Gravity waves from soft theorem in general dimensions, J. High Energy Phys. 09 (2018) 105.

[13] A. Laddha and A. Sen, Logarithmic terms in the soft expansion in four dimensions, J. High Energy Phys. 10 (2018) 056.

[14] In the analysis of Ref. [13], we had to make this assumption since there we ignored the contribution to the soft factor due to the outgoing radiation and therefore had to assume that the energy carried away by radiation is small compared to that carried by matter. Here, that assumption is not necessary since we shall explicitly take into account the contribution to the soft factor due to the outgoing radiation. Nevertheless, the approximation $M_{0} \simeq M$ is needed to ensure that we can regard the final configuration at late time as a set of light particles moving under the influence of the gravitational field of the stationary heavy object of mass $M_{0}$, ignoring the gravitational force exerted by the outgoing particles on each other. A more general formula where this assumption is not necessary can be derived using the general expression for the mutual gravitational force between the particles in relative motion [15].

[15] B. Sahoo and A. Sen, Classical and quantum results on logarithmic terms in the soft theorem in four dimensions, J. High Energy Phys. 02 (2019) 086.

[16] One word of caution may be added here. The general analysis of Ref. [12], on which this result is based, can determine $\tilde{e}_{i j}$ up to an overall phase that could depend on the energy $\omega$ but not on the polarization of the soft graviton. The phase given in (2) can be derived by comparing the general prediction of soft theorem with an explicit computation of the radiation during the scattering of a light particle from a heavy particle at large impact parameter [13]. We are assuming that the same phase can be used in this case as well. Since soft radiation comes from the domain where the light particles have moved sufficiently far away from the heavy center, we expect this relation to be valid. This is also in keeping with the results of effective field theory approach [17-19] where a similar phase has been found.

[17] W. D. Goldberger and A. Ross, Gravitational radiative corrections from effective field theory, Phys. Rev. D 81, 124015 (2010).

[18] R. A. Porto, A. Ross, and I.Z. Rothstein, Spin induced multipole moments for the gravitational wave amplitude from binary inspirals to 2.5 post-Newtonian order, J. Cosmol. Astropart. Phys. 09 (2012) 028.

[19] W. D. Goldberger, A. Ross, and I. Z. Rothstein, Black hole mass dynamics and renormalization group evolution, Phys. Rev. D 89, 124033 (2014).

[20] D. Christodoulou, Nonlinear Nature of Gravitation and Gravitational Wave Experiments, Phys. Rev. Lett. 67, 1486 (1991).

[21] K. S. Thorne, Gravitational-wave bursts with memory: The Christodoulou effect, Phys. Rev. D 45, 520 (1992).

[22] M. Favata, The gravitational-wave memory effect, Classical Quantum Gravity 27, 084036 (2010).

[23] A. Tolish and R. M. Wald, Retarded fields of null particles and the memory effect, Phys. Rev. D 89, 064008 (2014).

[24] L. Bieri and D. Garfinkle, Perturbative and gauge invariant treatment of gravitational wave memory, Phys. Rev. D 89, 084039 (2014).

[25] L. Blanchet and T. Damour, Hereditary effects in gravitational radiation, Phys. Rev. D 46, 4304 (1992).

[26] L. Blanchet and T. Damour, Post-Newtonian generation of gravitational waves, Ann. Inst. Henri Poincaré A 50, 377 (1989).

[27] L. Blanchet and G. Schaefer, Gravitational wave tails and binary star systems, Classical Quantum Gravity 10, 2699 (1993). 
[28] L. Blanchet, Gravitational wave tails of tails, Classical Quantum Gravity 15, 113 (1998); Erratum, Classical Quantum Gravity22, 3381(E) (2005).

[29] M. Favata, Post-Newtonian corrections to the gravitationalwave memory for quasi-circular, inspiralling compact binaries, Phys. Rev. D 80, 024002 (2009).

[30] M. Favata, Nonlinear gravitational-wave memory from binary black hole mergers, Astrophys. J. 696, L159 (2009).

[31] D. Pollney and C. Reisswig, Gravitational memory in binary black hole mergers, Astrophys. J. 732, L13 (2011).

[32] L. Blanchet and A. Le Tiec, First law of compact binary mechanics with gravitational-wave tails, Classical Quantum Gravity 34, 164001 (2017).
[33] P. C. Peters, Relativistic gravitational Bremsstrahlung., Phys. Rev. D 1, 1559 (1970).

[34] H. T. Janka, Explosion mechanisms of core-collapse supernovae, Annu. Rev. Nucl. Part. Sci. 62, 407 (2012).

[35] This computation was first done by Rishabh Batra as part of undergraduate research. This is only an order of magnitude estimate. Since the final state also contains other isotropically ejected matter, a full calculation requires the use of the general formula of Ref. [15] and a detailed model of the mass and velocity distribution of the final state particles.

[36] B. P. Abbott et al. (LIGO Scientific and Virgo Collaborations), Observation of Gravitational Waves from a Binary Black Hole Merger, Phys. Rev. Lett. 116, 061102 (2016). 